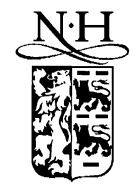

ELSEVIER

\title{
Radiation hardness and mechanical durability of Kuraray optical fibers
}

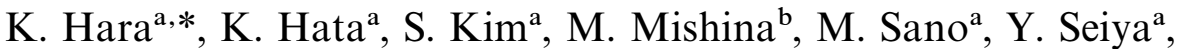 \\ K. Takikawa ${ }^{\mathrm{a}}$, M. Tanaka ${ }^{\mathrm{a}}$, K. Yasuoka ${ }^{\mathrm{a}}$ \\ a Institute of Physics, University of Tsukuba, Tsukuba-shi, Ibaraki-ken 305, Japan \\ ${ }^{\mathrm{b}}$ Fermi National Accerelator Laboratory, Batavia, IL 60510, USA
}

Received 8 October 1997

\begin{abstract}
The radiation hardness of Kuraray $3 \mathrm{HF}$ scintillating and clear optical fibers has been investigated using ${ }^{60} \mathrm{Co} \gamma$-rays in the dose range $0.4-500 \mathrm{krad}$. Significant initial degradation in the attenuation length was observed both for $3 \mathrm{HF}$ and clear fibers at a dose as small as $10 \mathrm{krad}$. The radiation hardness of both the scintillating and clear fibers is identical if it is expressed in terms of the ratio of the attenuation lengths after to before irradiation. The radiation damage of $3 \mathrm{HF}$ fibers was observed to recover substantially with a time scale of a few months. The attenuation length and mechanical durability against bending were measured for clear fibers by changing $S$ parameter which characterizes the softness of the fibers. (C) 1998 Elsevier Science B.V. All rights reserved.
\end{abstract}

PACS: 29.40. - n; 42.81.Bm; 42.88. + h

Keywords: Fiber tracker; Scintillating fiber; Radiation damage; Fiber transmittance

\section{Introduction}

Plastic scintillating and clear optical fibers have become one of the key detector elements in high energy experiments such as in fiber tracking systems and tile-fiber calorimeters. Radiation hardness remains to be an important issue for systems that have to be operated in high radiation environment. Ref. [1] reports that the attenuation length of

\footnotetext{
*Corresponding author. E-mail: hara@hep.px.tsukuba.ac.jp.
}

plastic fibers is shortened to about $60 \%$ of the original value after irradiation of $100 \mathrm{krad}$. Their measurement covers the dose range $100 \mathrm{krad}$ and above. We have carried out a series of radiation tests of Kuraray $3 \mathrm{HF}$ scintillating fibers and clear fibers to investigate the radiation hardness at smaller doses, extending the dose coverage down to $\sim 0.4 \mathrm{krad}$.

The attenuation length and mechanical durability against bending are also important parameters. This is of particular important for clear fibers of the tracker system where long clear fibers extract scintillating signals out of the detector complex 
through limited space. Kuraray has developed softer and more flexible fibers ( $S$ type) than the original type (or non- $S$ type) fibers for their applications to tile-fiber calorimeters where the wavelengthshifting fibers are bent at a radius of a few $\mathrm{cm}$ and embedded in scintillator plates [2]. The difference of $S$ and non- $S$ types is the degree ( $S$ parameter) of orientation of polystyrene chains, the core material of Kuraray's fibers. A modulation of diffractive index is sizable when orientation is well aligned, thus the attenuation length of $S$ type fibers can be shorter. In our previous report [3] we have verified that Kuraray $S$ type fibers are durable against bending at a bending radius as small as $\sim 1 \mathrm{~cm}$. In fiber tracker systems the bending radius of clear fibers could be designed to be somewhat larger. For the purpose of optimizing the $S$ value, we measured the attenuation length and mechanical durability of clear fibers with various $S$ values. In Kuraray's notation, S25 is the standard (non-S) type and S70 is the $S$ type fibers.

The present R\&D work has been carried out to design a fiber tracking system IFT [4] in the intermediate region of the CDF upgrade detector at the Fermilab Tevatron. The IFT consists of a set of cylindrical layers of $3 \mathrm{HF}$ scintillating fibers of 2 to $3 \mathrm{~m}$ length and covers the radial region from 20 to $40 \mathrm{~cm}$ around the beam pipe. The scintillating light is extracted outside the tracking volume through approximately $6 \mathrm{~m}$ long clear fibers. The expected radiation level to $3 \mathrm{HF}$ fibers can be estimated by extrapolating the radial dose profile measured with the CDF SVX' located in the range $3<r<$ $8 \mathrm{~cm}$ [5]. Extrapolating the dose profile to $r=$ $20(40) \mathrm{cm}$ gives a radiation level of $12(3.6) \mathrm{krad}$ per integrated luminosity of $1 \mathrm{fb}^{-1}$. The annual luminosity of the upgraded Tevatron will be $1 \mathrm{fb}^{-1}$ at beginning and $3 \mathrm{fb}^{-1}$ in later years.

The fibers used in the radiation hardness tests were Kuraray multiclad [6] S-type fibers. The diameter was $0.5-0.75 \mathrm{~mm}$ for $3 \mathrm{HF}(1 \%$ P-TP and $1500 \mathrm{ppm} 3 \mathrm{HF}$ ) fibers and $0.8 \mathrm{~mm}$ for clear fibers. The irradiation was performed at the ${ }^{60} \mathrm{Co}$ irradiation facility of Japan Atomic Energy Research Institute (JAERI), Tokai. Section 2 describes the procedure of irradiation and methods of radiation hardness evaluation. The results of the radiation hardness and its parameterization are given in Sec- tion 3. In Section 4 we describe the attenuation and mechanical durability of clear fibers as a function of the $S$ parameter. We measured the long-term stability of the attenuation of $S$ and non- $S$ type clear fibers, which is also given in Section 4 . The results are summarized in Section 5.

\section{Irradiation and evaluation of the radiation hardness}

\section{1. $3 H F$ fibers}

We have irradiated sample fibers at the JAERI ${ }^{60} \mathrm{Co} \gamma$-ray irradiation facility. A cylindrical source container of $9 \mathrm{~cm}$ radius is transported in the irradiation cave and placed on an exposure table of $\sim 80 \mathrm{~cm}$ in radius. The $\gamma$-ray intensity, calibrated with an ion chamber, is nearly proportional to $1 / r^{2}$ and is $4.0 \mathrm{kR} \mathrm{h}^{-1}$ at a distance of $70 \mathrm{~cm}$ for a source of $5.9 \mathrm{kCi}$ intensity. Thus, we can irradiate sample fibers uniformly by setting them at a certain radius around the source. The $\gamma$-ray intensity and uniformity were also measured with RCD dosimeter films [7] in the dose range $\sim 10 \mathrm{krad}$ and above, which is the usable range of the RCD films. These two measurements agreed with each other within $10 \%$.

The sample $3 \mathrm{HF}$ fibers were $3 \mathrm{~m}$ long and wrapped in aluminum foil at irradiation. The ${ }^{60} \mathrm{Co}$ irradiation took place twice for two different sets of samples. In the first irradiation $3 \mathrm{HF}$ fibers were placed at fixed distance of $30 \mathrm{~cm}$. These samples had a section near the fiber end shielded with $10 \mathrm{~cm}$ thick lead blocks. By measuring the light output continuity along the fiber we investigated the radiation damage to the $3 \mathrm{HF}$ wavelength shifter. In the second irradiation we set the samples at three radii 12,35 , and $75 \mathrm{~cm}$, and adjusted the exposure time so that the dose ranges at different radii overlapped each other. Using the overlapped data samples, we investigated the possible dose rate dependence and the mechanical damage due to different fiber curvatures at exposure.

The attenuation curve of $3 \mathrm{HF}$ fibers was measured by UV lamp scan with a system shown in Fig. 1. The sample fiber was strung by holding the ends, and a pencil type UV lamp was moved along 


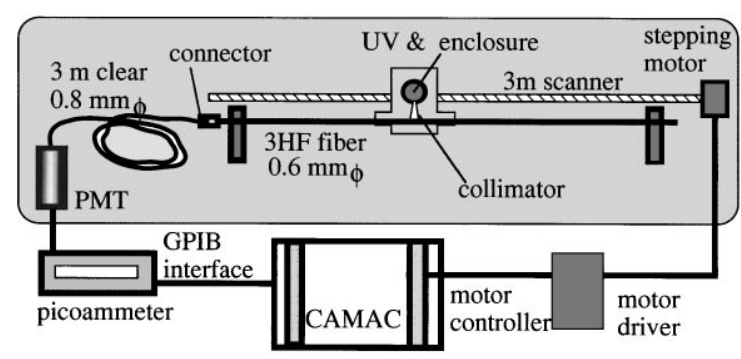

Fig. 1. Setup used to measure the attenuation length of $3 \mathrm{HF}$ fiber.

it using a stepping motor. A through hole was made in the UV lamp enclosure to guide and position the fiber precisely. The illumination spot of $\sim 5 \mathrm{~mm} \phi$ was determined by the collimator also machined in the enclosure. The light emerging from the sample $3 \mathrm{HF}$ fiber was transmitted through a $3 \mathrm{~m}$ long clear fiber of $0.8 \mathrm{~mm}$ diameter to a red-sensitive photomultiplier tube, Hamamatsu R2257. The quantum efficiency of this phototube is fairly flat in the entire $3 \mathrm{HF}$ emission region, $9 \%$ at $500 \mathrm{~nm}$ and $8 \%$ at $650 \mathrm{~nm}$. The shape of the emission spectrum could change by radiation damage due to wavelength dependence of the transmission degradation, see Section 3.3, use of a flat response PMT is essential in this measurement. The induced current was read out with a picoammeter, Keithley 485 . The scanning and data taking were computer controlled.

The UV lamp has an emission spectrum peaking at $360 \mathrm{~nm}$, which can excite the $3 \mathrm{HF}$ wavelength shifter but barely the primary flour P-TP. In order to evaluate the radiation damage to P-TP, we also irradiated four $3 \mathrm{HF}$ fiber ribbons, each consisting of staggered doublet layers of 16 of $0.5 \mathrm{~mm} \phi$ fibers arranged at a $0.6 \mathrm{~mm}$ pitch (Fig. 2), and the light yield was measured by $\beta$-source scan along the ribbon. Fig. 2 shows a cross-sectional view of a sample ribbon and a trigger system for ${ }^{90} \mathrm{Sr}$ $\beta$-rays. A ${ }^{90} \mathrm{Sr}$ source set above the sample and a trigger counter set underneath were moved together by a stepping motor. The light yield out of the ribbon was read out with a red-sensitive PMT through a $5 \mathrm{~m}$ long clear fiber ribbon. The charge induced by penetrating $\beta$-rays was measured with a CAMAC ADC. The light yields extrapolated to zero $3 \mathrm{HF}$ fiber length is sensitive to the damage to

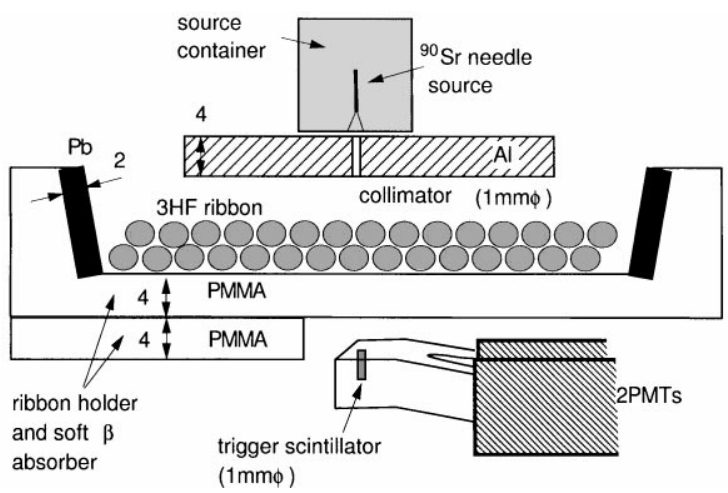

Fig. 2. Cross-sectional view of the light yield measurement system of a $3 \mathrm{HF}$ fiber ribbon. Penetrating $\beta$-rays are triggered by a scintillator viewed by two PMTs. Soft $\beta$-rays are absorbed by a $4 \mathrm{~mm}$ thick PMMA plate set underneath the sample $3 \mathrm{HF}$ ribbon.

the scintillating processes where both the primary (P-TP) and secondary (3HF) fluors are involved, since the effect from the attenuation degradation can be ignored. These samples were irradiated at a distance of $70 \mathrm{~cm}$ from the ${ }^{60} \mathrm{Co}$ source. One out of the four samples had its unread end mirrored with aluminum sputtering. The other samples had the unread end polished.

\subsection{Clear fibers}

We evaluated the attenuation length of clear fibers by two methods for two different dose ranges. In the higher dose range ( $>10 \mathrm{krad}$ ), we measured with a PMT the emerging light from the fiber end when $3 \mathrm{HF}$ light is injected from the other end of the clear fiber. For this measurement we used a Hamamatsu GaAs PMT R943-02, which had a quantum efficiency from $15 \%$ to $12 \%$ in the $3 \mathrm{HF}$ emission range. The clear fiber length, 5-6 $\mathrm{m}$ at the beginning, was shortened successively and this cut and measurement was repeated. Since the radiation hardness of clear fibers is dependent on the wavelength to be transmitted, we used a $3 \mathrm{HF}$ scintillating fiber excited by a UV lamp. In order to simulate the case where a charged particle traverses the middle of the IFT, the length between the illuminated point of the $3 \mathrm{HF}$ fiber and its end that couples to the clear fiber was set to $1 \mathrm{~m}$. The 
coupling between the $3 \mathrm{HF}$ and clear fibers was provided with a connector, which was not touched during the measurement. We shortened the clear fiber by $50 \mathrm{~cm}$ each time at the other end which coupled to the PMT. The cut end was polished with abrasive sheet set on a turning table. The light yield as a function of the clear fiber length can be expressed well by an exponential function, from which we extracted the attenuation length.

Since the accuracy of the attenuation length measurement with the above method was limited by the reproducibility of the fiber end preparation each time in shortening the clear fiber length, we carried out another test to cover the smaller dose range $(<2 \mathrm{krad})$ with a setup shown in Fig. 3. In this test the change of the light output was measured concurrently with ${ }^{60} \mathrm{Co}$ exposure, where we extracted the change of the attenuation length. We used a $0.8 \mathrm{~mm} \phi$ clear fiber of $5 \mathrm{~m}$ length, to which a $5 \mathrm{~cm}$ long $3 \mathrm{HF}$ fiber was connected. The $3 \mathrm{HF}$ fiber was illuminated by a UV lamp and the induced light was transmitted through the clear fiber to a PMT located outside the irradiation cave. Only a clear fiber section of $2 \mathrm{~m}$ length was exposed to ${ }^{60} \mathrm{Co}$ while the remaining part was shielded by lead blocks and the wall of the irradiation cave. The system of the $3 \mathrm{HF}$ fiber and the UV lamp were also shielded. The radiation damage to $3 \mathrm{HF}$ fibers is caused only through degradation in their attenuation length, as described in the next section. Therefore, even if the radiation to $3 \mathrm{HF}$ was not completely eliminated, we can ignore the damage to the $3 \mathrm{HF}$ light output by use of such a short $3 \mathrm{HF}$ fiber. The stability of the UV lamp and 3HF light source was monitored using a quartz fiber.

\section{Results of the radiation hardness}

\subsection{Evaluation of the dose}

In $\gamma$-ray exposure the energy is deposited mainly by Compton electrons created by ${ }^{60} \mathrm{Co} \gamma$ 's of $\sim 1.2 \mathrm{MeV}$. Since some fraction of Compton electrons can escape from the sample fiber due to its finite volume, the dose is dependent on the geometry of the sample. The translation factor from Roentgen, given as a function of the distance from

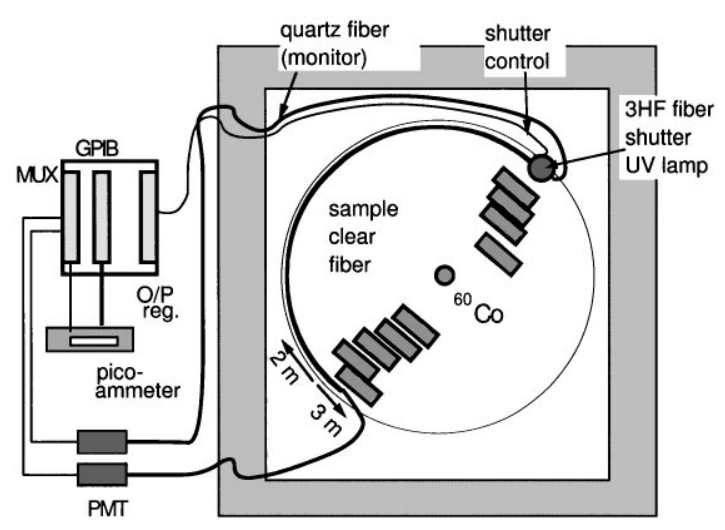

Fig. 3. Setup used to measure the attenuation length change of a clear fiber at irradiation.

the source and the exposure time, to the absorbed dose was calculated using a GEANT simulation. In order to check the reliability of the simulation, we exposed scintillating fiber ribbons of different thicknesses, $0.5,0.75$, and $1.5 \mathrm{~mm} \phi$, to a ${ }^{60} \mathrm{Co}$ source, and measured the induced current. The measured ratio of the current normalized by volume out of the $0.5 \mathrm{~mm} \phi$ fiber ribbon to that out of the $1.5 \mathrm{~mm} \phi$ fiber ribbon was $0.85 \pm 0.04$, while we obtained a simulated ratio of $0.80 \pm 0.03$. The simulation can provide results consistent with the experimental results. The Roentgen-to-dose translation factor for single fibers was extracted from simulation. With use of $\mathrm{Al}$ foils as the wrapping material, the translation factor is made almost constant within 0.6 to 0.7 for fibers of diameter smaller than $1 \mathrm{~mm}$. We hereafter take a translation factor of 0.65 for single fibers. The overall uncertainty of the dose is about $20 \%$.

\subsection{Radiation hardness of $3 H F$ fibers}

Fig. 4 shows an example of the $3 \mathrm{HF}$ attenuation curves measured as a function of the UV lamp position, $x$. The fiber was irradiated to $48 \mathrm{krad}$ at $12 \mathrm{kradh}^{-1}$ uniformly along the fiber except the part $x<45 \mathrm{~cm}$, which was shielded by lead blocks. The light yields are normalized by the average of the three data points at $x=20,30$ and $40 \mathrm{~cm}$. The four curves in the figure are those measured before, $2 \mathrm{~d}$ after, $13 \mathrm{~d}$ after and $260 \mathrm{~d}$ after the irradiation. 


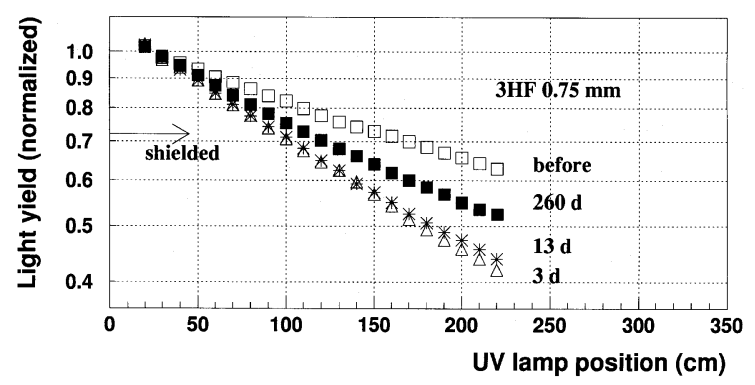

Fig. 4. Attenuation curves of a $3 \mathrm{HF}$ fiber measured before (open squares), $3 \mathrm{~d}$ (triangles) after, $13 \mathrm{~d}$ after (crosses), and $260 \mathrm{~d}$ after (filled squares) the irradiation. The dose was $48 \mathrm{krad} \mathrm{ex}-$ cluding the part from 0 to $45 \mathrm{~cm}$ which was shielded by lead blocks.

Since no discontinuity is visible for the irradiated sample across the position $x=45 \mathrm{~cm}$, we conclude that $3 \mathrm{HF}$ scintillation mechanism itself is not destroyed up to this dose. Instead, the attenuation length is shortened; fitting the curves in $x>50 \mathrm{~cm}$ gives the attenuation length 4.5 and $2.45 \mathrm{~m}$ before and $13 \mathrm{~d}$ after the irradiation, respectively. The attenuation length gradually recovered as is evident from the figure, which will be discussed in Section 3.4.

Measurements of doublet fiber ribbons using penetrating $\beta$-rays are summarized in Table 1 . The damage to the light yield is small at positions close to $x=0$ as shown in the table. Extrapolations to $x=0$ are consistent with no damage within the measurement uncertainty for the samples with no mirror. The sample with mirror showed some deviation from unity, which is due to that the light component reflected by the mirror is reduced by the attenuation length degradation. The present results support the previously obtained conclusion [8] that both of the scintillating dyes P-TP and $3 \mathrm{HF}$ are not damaged in the dose range under study.

In Fig. 5 we plot the ratios of the attenuation lengths after to before the irradiation for $3 \mathrm{HF}$ fibers and $3 \mathrm{HF}$ fiber ribbons. The measurements were made 1-2 weeks after the irradiation. Also plotted are results for clear fibers, which are described in the following section.

We expect that damage to $3 \mathrm{HF}$ fibers and to clear fibers are similar if the damage is expressed by the ratio of the attenuation lengths before to after irradiation, since the damage to both fibers occurs through degradation in the attenuation length. In fact, the data points in Fig. 5 seem to lie on a single line:

$$
\begin{aligned}
& \lambda / \lambda_{0}=(0.80 \pm 0.01)-(0.144 \pm 0.007) \log _{10} D: \\
& D>0.1 \mathrm{krad}
\end{aligned}
$$

where $D$ is the dose in $\mathrm{krad}$ and $\lambda_{0}$ is the attenuation length with no damage.

We note that the $3 \mathrm{HF}$ fiber samples exposed at different distances from the ${ }^{60} \mathrm{Co}$ source, $12-75 \mathrm{~cm}$, do not significantly deviate from each other. Therefore we expect there should be no significant improvement in the radiation hardness when fibers are kept straight at irradiation, which is relevant for fibers used in trackers.

\subsection{Radiation hardness of clear fibers}

Radiation hardness of clear fibers, plotted in Fig. 5, is similar to that of $3 \mathrm{HF}$ fibers as far as

Table 1

\begin{tabular}{|c|c|c|c|c|}
\hline & \multirow{2}{*}{$\frac{47 \mathrm{krad}}{\mathrm{w} / \mathrm{o} \mathrm{mirror}}$} & \multicolumn{2}{|l|}{$380 \mathrm{krad}$} & \multirow{2}{*}{$\frac{680 \mathrm{krad}}{\mathrm{w} / \mathrm{o} \mathrm{mirror}}$} \\
\hline & & w/o mirror & $\mathrm{w} /$ mirror & \\
\hline Light yield ratio at $0.1 \mathrm{~m}$ & $0.97 \pm 0.07$ & $0.96 \pm 0.04$ & $0.86 \pm 0.08$ & $0.95 \pm 0.05$ \\
\hline Light yield ratio at $1.1 \mathrm{~m}$ & $0.88 \pm 0.07$ & $0.70 \pm 0.03$ & $0.69 \pm 0.07$ & $0.67 \pm 0.03$ \\
\hline Light yield ratio at $2.1 \mathrm{~m}$ & $0.77 \pm 0.06$ & $0.64 \pm 0.02$ & $0.63 \pm 0.04$ & $0.53 \pm 0.02$ \\
\hline Attenuation length ratio & $0.73 \pm 0.07$ & $0.59 \pm 0.03$ & $0.43 \pm 0.08$ & $0.40 \pm 0.02$ \\
\hline
\end{tabular}

Ratio of the light yields and the attenuation lengths after to before the irradiation, measured for $3 \mathrm{HF}$ ribbons

Note: One of the four samples has aluminum mirror at the unread fiber ends. 


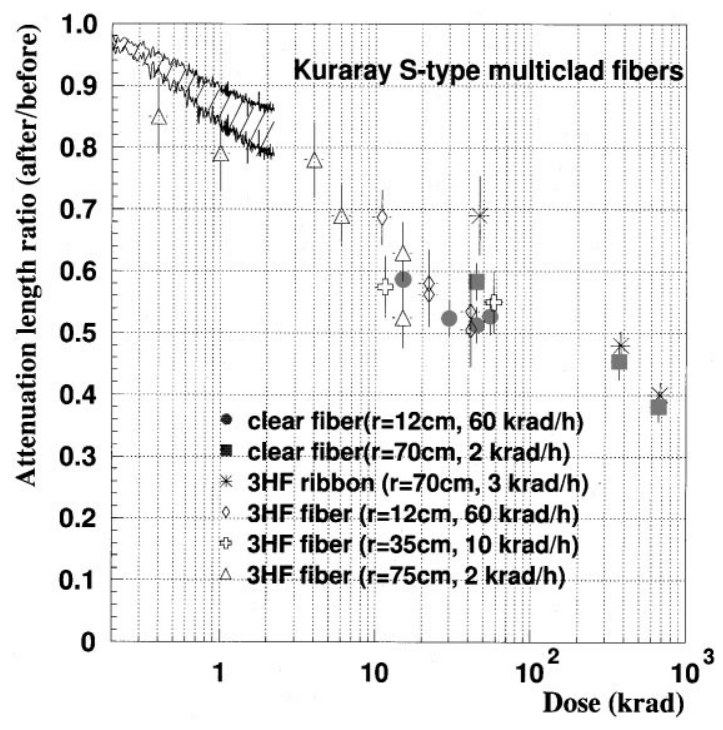

Fig. 5. Ratio of the attenuation lengths before to after irradiation. The samples are $3 \mathrm{HF}$ fibers (open marks), $3 \mathrm{HF}$ fiber ribbons (crosses), and clear fibers (filled marks). The fiber curvature at irradiation and the dose rate are given in parentheses. The shaded region shows the range of the ratio for a clear fiber where the attenuation length was evaluated concurrently with irradiation at $2 \mathrm{kradh}^{-1}$ (see text). Other data were measured 1-2 weeks after the irradiation.

the ratio of the attenuation lengths after to before the irradiation is compared. In Fig. 5 the drop of the clear fiber attenuation length measured concurrently with the irradiation is also shown by the shaded region. This sample was irradiated at a rate of $2 \mathrm{kradh}^{-1}$. Assuming an exponential attenuation in the clear fiber, one can extract the ratio of the attenuation lengths during to before the irradiation through the relation:

$\lambda / \lambda_{0}=\left(\frac{\lambda_{0}}{l_{0}} \ln \frac{L_{0}}{L}+1\right)^{-1}$,

where $\lambda / \lambda_{0}$ is the ratio of the attenuation lengths during to before irradiation, $l_{0}$ is the length of exposed part ( $2 \mathrm{~m})$, and $L / L_{0}$ is the ratio of the light outputs. From the measurement of $L / L_{0}$, the ratio of the attenuation lengths can be calculated. We measured the attenuation lengths of the irradiated and shielded sections separately after the irradiation. We obtained $\lambda_{0}=6.6 \mathrm{~m}$ for the shielded section, which is taken in the calculation. The shaded region corresponds to the systematic uncertainties. Among these the contribution from the instability of the $3 \mathrm{HF}$ light source (the UV lamp instability and the $3 \mathrm{HF}$ damage induced by the UV lamp and irradiation) was evaluated to be at most $5 \%$ where the main cause of the instability was the temperature variation. We recognized that the output current changed by $2-3 \%$ in a minute right after the source was removed. The systematic uncertainties include also this short time light output variation and the uncertainty in $\lambda_{0}$.

As described before we used a $3 \mathrm{HF}$ scintillating fiber as light source for attenuation measurements of clear fibers, since the absorption is dependent on the wavelength of the light to be transmitted. Fig. 6 shows the absorption spectra of a unirradiated and two irradiated samples. The irradiated samples were measured eight days after the irradiation. The absorption was measured with an optical spectrum analyzer, Anritsu MS9701, and was defined as the ratio of the light yield from a $6 \mathrm{~m}$ clear fiber and that from a $1 \mathrm{~m}$ fiber. The attenuation length $\lambda$ is thus related to the absorption $A$ through

$\lambda(\mathrm{m})=5(\mathrm{~m}) / \ln \left(10^{A / 10}\right)=21.7(\mathrm{~m}) / A$.

Hence, $\lambda=10.8$ (5.4) $\mathrm{m}$ for $A=2$ (4) dB/5 m. Larger absorption in shorter wavelengths is due to Rayleigh scattering. The peaks in the spectrum are characteristic absorption bands of polystyrene molecule. The damage to the attenuation length is larger at shorter wavelengths and is negligibly small at wavelengths longer than $700 \mathrm{~nm}$. Note that the $3 \mathrm{HF}$ emission spectrum ranges from 500 to $650 \mathrm{~nm}$ with the peak at $530 \mathrm{~nm}$.

\subsection{Recovery of the radiation damage}

The expected dose rate in the IFT is at most $\sim 2 \mathrm{radh}^{-1}$, while we irradiated the samples at accelerated rates of 2-60 $\mathrm{krad} \mathrm{h}^{-1}$. Ref. [1] reports that the attenuation length of $3 \mathrm{HF}$ fibers irradiated to $140 \mathrm{krad}$ at $16 \mathrm{krad} \mathrm{h}^{-1}$ showed a drop substantially (to $20 \%$ or less of the original length) right after the irradiation but recovered to $\sim 50 \%$ in $\sim 10 \mathrm{~h}$. The recovery was traced for a couple of days, and seems to be stable in this period. Another group [9] has studied the recovery of the attenuation length of fibers exposed to ambient 


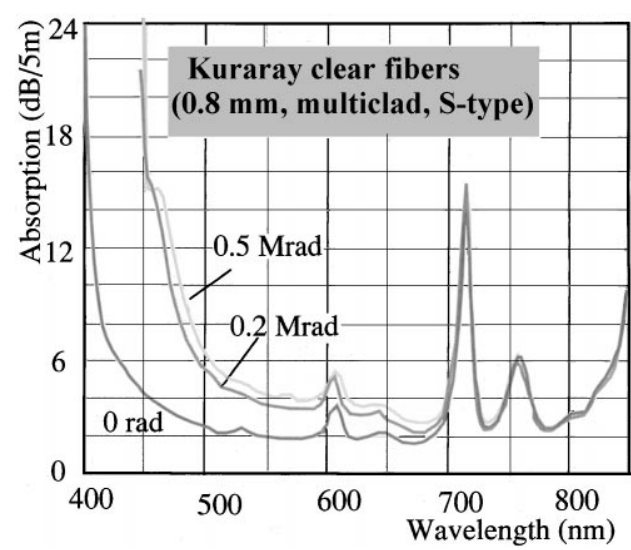

Fig. 6. Absorption spectra of clear fibers unirradiated, irradiated to $0.2 \mathrm{Mrad}$, and irradiated to $0.5 \mathrm{Mrad}$.

fluorescence light and reports that the damage is recovered completely in 5-20 d. The radiation damage is explained by creation of free radicals, which act as color centers and degrade the light transmission. The recovery is the process where oxygen reacts with such free radicals yielding oxidation products or thermal annealing is taking place [8]. The radiation-induced coloration thus starts to disappear after irradiation and the transmittance is recovered, but such oxidation products can degrade the transmittance [8], which is one of the causes for the permanent damage in the attenuation length.

Fig. 7 plots the recovery of the attenuation length of $3 \mathrm{HF}$ fibers measured in the period up to $260 \mathrm{~d}$ after the irradiation. The samples were kept in the air at room temperature and shielded against ambient light. Although the damage measured $3 \mathrm{~d}$ after the irradiation is dependent on the accumulated dose, the recovery seems to occur similarly for the doses we tested. Also the recovery rate seems similar for the two dose rates 2 and $12 \mathrm{krad} \mathrm{h}^{-1}$ we measured. The ratio of the attenuation length can be expressed approximately by

$$
\lambda(T) / \lambda_{0}=a_{0}+(9-12) \% \log _{10}(T): \quad T>3
$$

where $a_{0}$ is the ratio measured $3 \mathrm{~d}$ after the irradiation and $T$ is in days.

Such a slow recovery is also reported in Ref. [10]. In Ref. [10], 3HF and clear fibers (Kuraray multi-

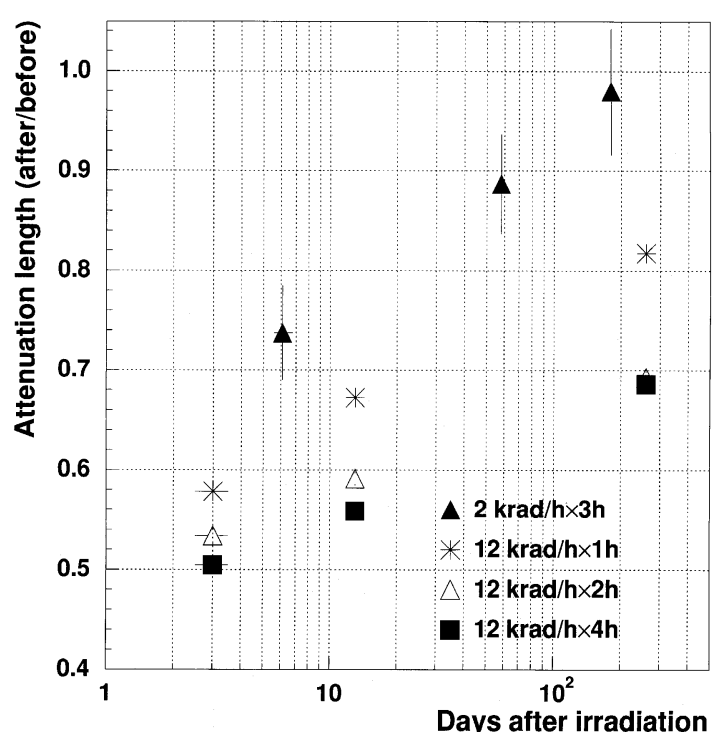

Fig. 7. Recovery of the attenuation length of $3 \mathrm{HF}$ fibers, irradiated at two different dose rates and to four different doses. Typical size of the systematic uncertainty is indicated for the sample irradiated at $2 \mathrm{kradh}^{-1}$.

clad fibers) were irradiated by a ${ }^{137} \mathrm{Cs}$ source at $\sim 20 \mathrm{krad} \mathrm{h}^{-1}$ to $0.5 \mathrm{Mrad}$. The light yield out of $1 \mathrm{~m}$ long $3 \mathrm{HF}$ fibers was $\sim 20 \%$ of the original light yield just after irradiation, $\sim 20 \%$ after $20 \mathrm{~d}$ and 55-90\% (varies between samples) after two months, while the light output out of clear fibers was nearly constant at $30-50 \%$ during the same period. By assuming an exponential attenuation in $3 \mathrm{HF}$ fibers and $\lambda_{0} \sim 3 \mathrm{~m}$, the initial damage of $20 \%$ of the original light yield can be translated into degradation in the attenuation length of $\lambda / \lambda_{0} \sim 0.17$, which should be compared with our ratio of $\sim 0.4$ at $0.5 \mathrm{Mrad}$. Although the magnitude of the initial damage seems not identical, the tendency of the recovery is roughly consistent with each other. We have no data available for clear fibers, because of our destructive tests and we did not keep extra fibers.

Because of existence of a slow recovery component, evaluation of the detector performance in the real radiation environment is not straightforward. We have carried out a GEANT simulation to evaluate the dose profile along the $3 \mathrm{HF}$ and clear fibers of the IFT. Using the damage parameterization Eq. (1), the light yield is calculated to decrease 
by $38-35 \%(48-45 \%)$ of the original light yield after a delivered luminosity of $3(10) \mathrm{fb}^{-1}$, where the range of the numbers corresponds to the different position of the $3 \mathrm{H}$ fibers from the beam pipe. Of these drops about $1 / 4$ is due to the clear fiber and the rest is due to the $3 \mathrm{HF}$ fiber. The actual damage during the experiment may have to be evaluated through irradiation tests at much lower dose rates where the recovery process should be taking place concurrently with damaging process. Here we evaluate the impact of a year long break of operation scheduled after $3 \mathrm{fb}^{-1}$. The light yield decreases by $38-35 \%$ if we assume no recovery during the experiment, as mentioned above. This drop will recover according to Eq. (4), resulting a smaller drop of $21-14 \%$ if only $3 \mathrm{HF}$ is assumed to recover.

\section{Durability of the clear fibers}

\subsection{Attenuation length dependence on the $S$ parameter}

The $S$ parameter is a characteristic index of the degree of orientation of polystyrene chains along the fiber axis. At larger $S$ values polystyrene chains are aligned and the fibers can become softer and more flexible. On the other hand, the attenuation length can be shorter due to possible modulation of diffractive index values along the fiber. An extreme case of larger $S$ values would be the fishing string which is strong and flexible but is not transparent.

We have changed the parameter $S$ from S25 (non- $S$ type) to $S 70$ ( $S$ type) and measured the attenuation length. A set of sample fibers were fabricated from the same preform, therefore we can ignore the individual difference otherwise arising due to different preform. Fig. 8 shows the attenuation lengths as a function of the $S$ parameter, the attenuation length being measured for 1-4 samples at each $S$ value. The attenuation length decreases with $S$. For example, the attenuation length of $S 40$ is typically $12 \mathrm{~m}$, while that of S70 is $9 \mathrm{~m}$. Since $\mathrm{S} 40$ is substantially superior over S25 and S30 in view of mechanical durability as described below, we have carried out another comparison measurement between S40 and S70 where we measured the emerging light yield when $3 \mathrm{HF}$ light was injected

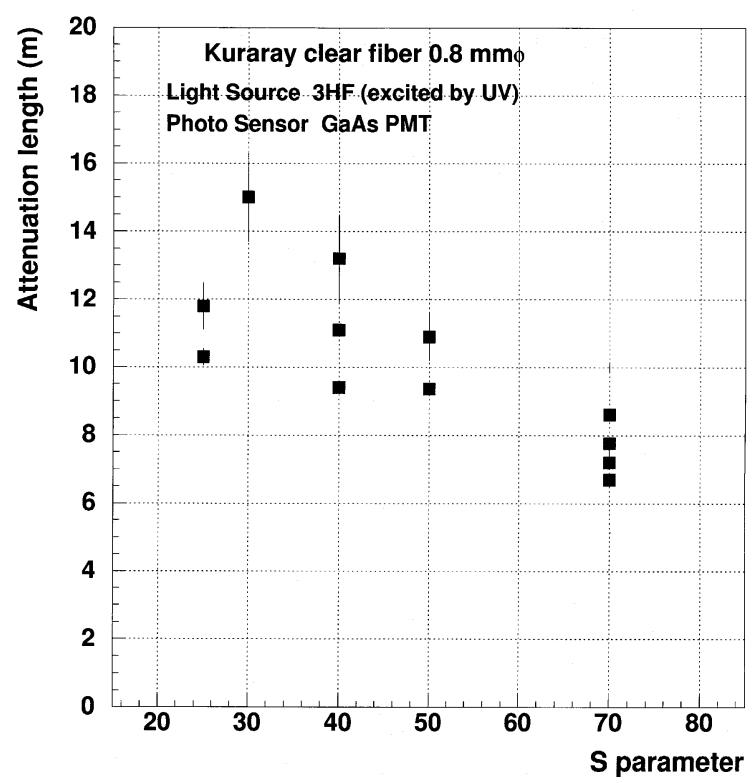

Fig. 8. Attenuation length of $0.8 \mathrm{~mm} \phi$ clear fibers as a function of $S$ parameter. The light source is $3 \mathrm{HF}$ scintillating fiber excited by a UV lamp. Typical size of measurement uncertainty is indicated for some of the data.

from the other end of $6 \mathrm{~m}$ long samples. From the average of 10 samples each for S40 and S70, the light yield out of S40 fibers was larger by $16 \%$ than that out of S70 fibers, which is consistent with the improvement expected from the longer attenuation length of S40 fibers.

\subsection{Transmittance degradation at bending}

In order to qualify the mechanical durability against bending, we measured the transmittance of sample clear fibers rolled around plastic tubes of $0.5,1$ and $2 \mathrm{~cm}$ in radius. The number of turns were 20, 16 and 16, respectively. In this measurement the samples were coupled to green LEDs and the emerging light yields were measured by a common PMT. By switching the LEDs the transmittance of the corresponding fiber was traced for over two months. The possible variation of the LED intensity was monitored by another PMT.

Fig. 9 plots the stability of the light output for fibers rolled at radii of 1 and $2 \mathrm{~cm}$. The data are shown only for S25 (non-S), S30 and S40, since 


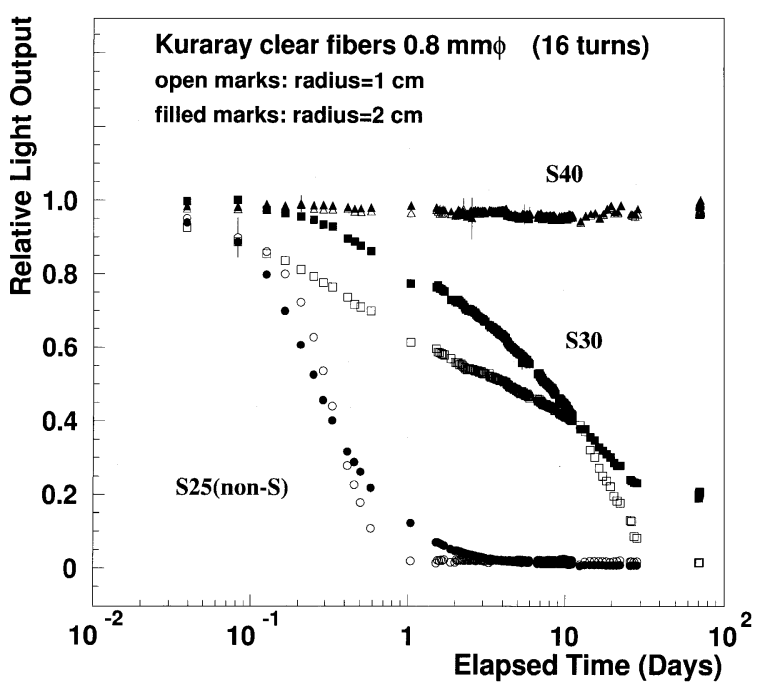

Fig. 9. Light output as a function of elapsed time since fibers were bent. The fibers were rolled by 16 turns at a radius of $1 \mathrm{~cm}$ (open marks) and of $2 \mathrm{~cm}$ (filled marks).

S50-S70 showed stable light output similar to S40. The light output of S25 started to drop in an hour and went down to almost zero in a day, while that of S40 was stable during this period of measurement. At a bending radius of $0.5 \mathrm{~cm}$, the fibers $\mathrm{S} 40$ through S60 also showed a substantial drop just after they were rolled, although the tendency that the fibers are more flexible for larger $S$ values is still valid. From these measurements we conclude that S40 is substantially durable against bending in comparison with S30 and S25 as long as the bending radius is kept larger than $\sim 1 \mathrm{~cm}$.

\subsection{Long term stability of clear fiber attenuation length}

The long term stability of the clear fiber transmittance is an interesting issue to pursue from the long term stability point of view of the detector performance. It is also important for the way of storing the fiber whether the fiber has to be kept straight or can be reeled. We measured periodically the attenuation length for almost three years both for $S$ and non- $S$ type fibers of $0.8 \mathrm{~mm} \phi$ thickness. The fibers have been rolled on reels of $50 \mathrm{~cm}$ in radius since the production. The reels have been

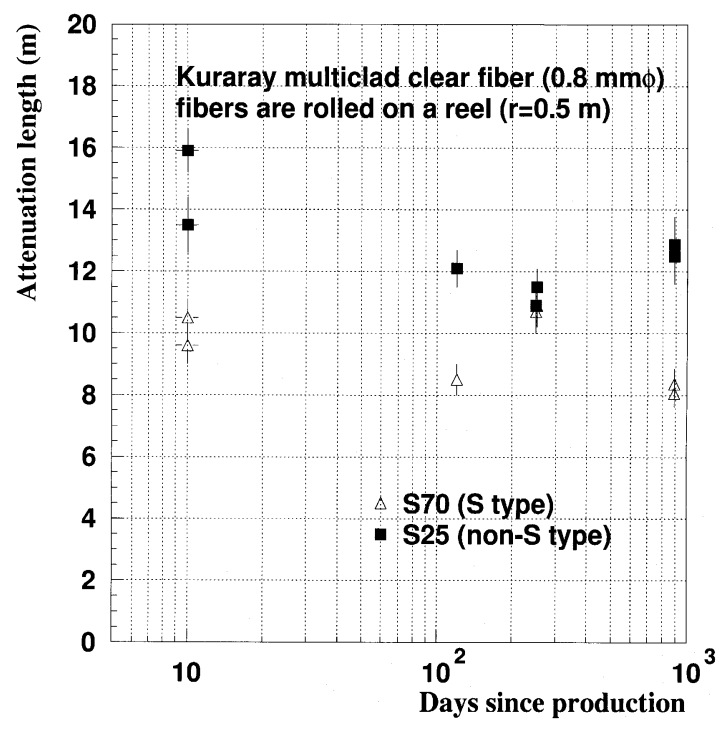

Fig. 10. Attenuation length of clear fibers as a function of elapsed time since production. The fibers, $S$ and non- $S$ types, were rolled on reels of $0.5 \mathrm{~m}$ in radius.

contained in a wooden box, which has been kept in our laboratory at room temperature. Although the fibers have never been exposed to intense light such as direct rays of the sun, the fibers were exposed to ambient room light in the period for $\sim 200 \mathrm{~d}$ after production where the lid of the wooden box was open: after that the fibers were kept in dark.

The results are plotted in Fig. 10. The number of samples was typically two for each of $S$ and non- $S$ type fibers. The attenuation length is fairly stable in this period of three years since production. The attenuation length may slightly decrease from that right after the production, which could be attributed to effect of ambient room light described above or to any other initial effect that shortens the attenuation length of new fibers. The attenuation lengths are approximately 12 and $8 \mathrm{~m}$ for non- $S$ and $S$ type fibers even after three years.

\section{Summary}

We have measured the radiation hardness of Kuraray $3 \mathrm{HF}$ scintillating and clear fibers in the dose range from $\sim 0.4 \mathrm{krad}$ to $0.5 \mathrm{Mrad}$, and 
studied the optical and mechanical properties of clear fibers with various $S$ values. The ratio of the attenuation lengths before and after the irradiation is similar for both $3 \mathrm{HF}$ and clear fibers. The damage function derived from the data measured 1-2 weeks after the irradiation is given in this note. The damage of $3 \mathrm{HF}$ fibers showed a substantial recovery with a time scale of a few months. The recovery rate is rather insensitive to the dose rate $2-12 \mathrm{kradh}^{-1}$ and to the accumulated dose of 6-48 krad we tested. We have shown that the attenuation length of clear fibers is longer for smaller $S$ values. For a bending radius of $>1 \mathrm{~cm}, \mathrm{~S} 40$ seems to be a good compromise, which is mechanically durable and the attenuation length is longer by $\sim 30 \%$ than $S$-type (S70) fibers. The attenuation length of clear fibers which have been reeled at a radius of $50 \mathrm{~cm}$ was traced for three years. The attenuation length turned out to be fairly stable in this period.

\section{Acknowledgements}

We would like to thank O. Shinji (Kuraray) for preparing the sample fibers and $H$. Nagayama (JAERI) for carrying out ${ }^{60} \mathrm{Co}$ irradiation. This work was supported by the Japanese-US cooperation in the field of High Energy Physics.

\section{References}

[1] S. Margulies et al., in: A.D. Bross, R.C. Ruchti, M.R. Wayne (Eds.), Proc. SCFI93 Workshop on Scintillating Fiber Detectors, Notre Dame, World Scientific, Singapore, 1995, p. 421.

[2] See, for example, CDF Plug Upgrade Tile/Fiber Calorimeter, The CDF II Detector Technical Design Report, FERMILAB-Pub-96/390-E, 1996.

[3] K. Hara et al., Nucl. Instr. and Meth. A 373 (1996) 347.

[4] Proposal for RUN II Tracking System Upgrades for CDF, CDF/TRACKING/PUBLIC/3079 (1995). This fiber system, however, was not adopted as the intermediate tracking device for CDF.

[5] D. Amidei et al., Nucl. Instr. and Meth. A 350 (1994) 73; P. Azzi et al., CDFnote 3278. The dose measured with a dosimeter was $300 \mathrm{rad} / \mathrm{pb}^{-1}$ for the SVX layer 0 $(r=3 \mathrm{~cm})$. The radial dependence $r^{-1.7}$ was obtained from the increases in the leakage current measured for the layers 0 to $3(3<r<8 \mathrm{~cm})$.

[6] Multiclad fibers have two cladding layers of PMMA $(n=1.49)$ and fluorinated MMA $(n=1.42)$ surrounding a polystyrene core $(n=1.59)$. Existence of the second clad makes high the light trapping efficiency. See S. Aota et al., Nucl. Instr. and Meth. A 357 (1995) 71.

[7] Far West Technologies, Goleta, CA, USA.

[8] See, for example, A.D. Bross, A. Pla-Dalmau, Nucl. Instr. and Meth. A 327 (1993) 337; A.D. Bross, A. Pla-Dalmau, IEEE Trans. Nucl. Sci. NS-39 (1992) 1199.

[9] M. Chung, S. Margulies, in: A.D. Bross, R.C. Ruchti, M.R. Wayne (Eds.), Proc. SCFI93 Workshop on Scintillating Fiber Detectors, Notre Dame, World Scientific, Singapore, 1995, p. 431.

[10] F. Bedeschi et al., CDF note 3583. 